\title{
Chronic Total Coronary Artery Occlusion
}

National Cancer Institute

\section{Source}

National Cancer Institute. Chronic Total Coronary Artery Occlusion. NCI Thesaurus. Code C100062.

Prolonged complete obstruction of the coronary artery. (ACC) 